\section{§5. Spectroscopic Study of Carbon Pellet Ablation Cloud}

Goto, M., Morita, S.

Carbon pellets were injected into the plasma and radiation from the ablation cloud in the UV-visible wavelength range was observed. The observed spectrum is found to be dominated by emission lines of CII and CIII ions and populations of the corresponding excited levels are determined from the measured line intensities. The result shown in Fig. 1 suggests establishment of LTE (local thermodynamic equilibrium) over the measured excited levels of the both ions. On

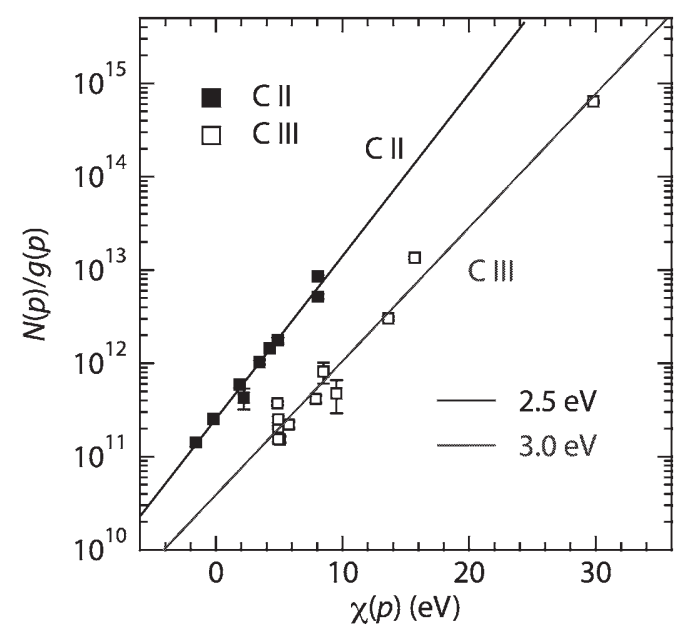

Fig. 1: Volume-integrated populations of excited levels normalized by their statistical weight for CII (solid squares) and CIII (open squares) ions.

the assumption that level populations follow the Boltzmann distribution the electron temperature $T_{\mathrm{e}}$ is determined to be $2.5 \mathrm{eV}$ and $3.0 \mathrm{eV}$ from a fit of the measured CII and CIII populations, respectively. The fitting results are shown in Fig. 1 with solid lines. We consider a two-layer model for the ablation cloud: the plasma which dominantly emits CII lines is enclosed by another plasma which is responsible for the observed CIII lines. The former and latter are hereafter called the $\mathrm{CII}$ and CIII plasmas, respectively.

For the CII plasma the electron density, $n_{\mathrm{e}}=6.5 \times$ $10^{22} \mathrm{~m}^{-3}$, and plasma volume, $V=5.3 \times 10^{-6} \mathrm{~m}^{3}$, are simultaneously derived from a fit of the CII $\lambda 723 \mathrm{~nm}$ $\left(\left[1 s^{2} 2 s^{2}\right] 3 p^{2} P^{o}-3 d^{2} D\right)$ line profile which is shown in Fig. 2 with the open squares. In this fitting, Stark broadening, Doppler broadening, Zeeman effect, and the reabsorption effect are taken into account. The fitting result is shown in Fig. 2 with the black solid line. The vertical bars indicate the Zeeman split components for the magnetic field strength of 2.0 $T$. The grey dashed and grey solid lines show the Gaussian profile due to the Doppler broadening and the convolution of the Doppler and Stark broadening profiles, respectively. The instrumental function is convoluted for all the synthetic profiles.

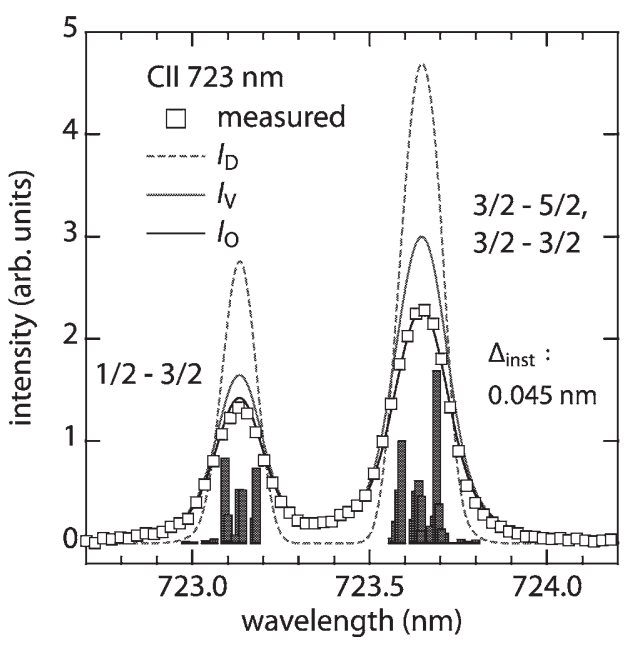

Fig. 2: Emission line profile of the CII $\left[1 \mathrm{~s}^{2} 2 \mathrm{~s}^{2}\right] 3 \mathrm{p}^{2} \mathrm{P}^{\mathrm{o}}-$ $3 \mathrm{~d}^{2} \mathrm{D}$ lines (open squares) and fitting results (lines).

For the CIII plasma observation of Stark broadening has been also attempted for various lines only to find no significant broadening. A clue to $n_{\mathrm{e}}$ determination is found instead in a set of CIII fine structure lines in the VUV wavelength range $(\lambda \sim 117 \mathrm{~nm})$ which correspond to the transitions of $\left[1 \mathrm{~s}^{2}\right] 2 \mathrm{~s} 2 \mathrm{p}^{3} \mathrm{P}_{0,1,2}^{\mathrm{o}}-2 \mathrm{p}^{2}{ }^{3} \mathrm{P}_{0,1,2}$. The apparent population ratios among the upper fine structure levels are found to considerably deviate from those of the corresponding statistical weights, which may be caused by the reabsorption effect. We have assumed a spherical plasma and have evaluated the escape factors for each of the fine structure lines. This result and the absolute population of the lower level of the transition, which has been derived from the UV-visible range measurement and the assumption of Boltzman equilibrium, gives the plasma volume of $V=4.5 \times 10^{-2} \mathrm{~m}^{3}$. Furthermore, an assumption of electric charge neutrality in the ablation cloud yields $n_{\mathrm{e}}=4.7 \times 10^{20} \mathrm{~m}^{-3}$.

We have assumed establishment of complete LTE over all the charge state ions to determine $n_{\mathrm{e}}$ and $V$ in the analyses above. Although this assumption is found to be acceptable for the CII plasma, the value of $n_{\mathrm{e}}$ for the CIII plasma seems to be too low for CIII levels to be in complete LTE. The validity of complete LTE has been examined for CIII levels in the CIII plasma. To this end we have developed a collisionalradiative model, in which reabsorption effects are taken into account. When the reabsorption effect is significant, it is confirmed that the CIII populations approach the LTE values and the assumption of complete LTE is acceptable. The parameters obtained for the CII and CIII plasmas are tabulated in Table I.

Table I: Parameters obtained for the CII and CIII plasmas.

\begin{tabular}{llll}
\hline \hline & $T_{\mathrm{e}}(\mathrm{eV})$ & $n_{\mathrm{e}}\left(\mathrm{m}^{-3}\right)$ & $V\left(\mathrm{~m}^{3}\right)$ \\
\hline CII & 2.5 & $6.5 \times 10^{22}$ & $5.3 \times 10^{-6}$ \\
CIII & 3.0 & $4.7 \times 10^{20}$ & $4.5 \times 10^{-2}$ \\
\hline \hline
\end{tabular}

